\title{
ANWENDUNGEN EINER METHODE VON PÓLYA IN DER THEORIE DER GANZEN FUNKTIONEN
}

\author{
LUTZ VOLKMANN
}

\section{Bezeichnungen und einleitende Bemerkungen}

1.1. Es seien $f$ eine ganze Funktion, $M(r, f)=M(r)$ der Maximalbetrag, $m(r, f)=m(r)$ der Minimalbetrag, $v(r, f)=v(r)$ der Zentralindex, $n(r, 0, f)=$ $n(r, 0)$ die Anzahl der Nullstellen von $f$ im Kreis $|z| \leqq r, N(r, 0, f)=N(r, 0)$ die Nevanlinnasche Anzahlfunktion, $T(r, f)=T(r)$ die Nevanlinnasche Charakteristik und

$$
\bar{N}(r, 0, f)=\bar{N}(r, 0)=\int_{0}^{r} \frac{N(t, 0)-n(0,0) \log t}{t} d t+\frac{1}{2} n(0,0) \log ^{2} r .
$$

Mit

$$
\varrho=\varrho(f)=\limsup _{r \rightarrow \infty} \frac{\log \log M(r)}{\log r}
$$

wird die Ordnung von $f$ bezeichnet.

1.2. Im Fall, daß $f$ eine ganze Funktion der Ordnung $\varrho<1$ ist, haben Valiron [14] und Wiman [19] unabhängig voneinander den bekannten, von Littlewood [8] vermuteten $\cos \pi \varrho$-Satz bewiesen

$$
\limsup _{r \rightarrow \infty} \frac{\log m(r)}{\log M(r)} \geqq \cos \pi \varrho .
$$

Später gab Pólya [12] einen neuen und eleganten Beweis von (1.1), wobei das Theorem II in dieser Arbeit das zentrale Ergebnis darstelit. Diese Methode von Pólya benutzten Ostrovskiǐ [10] sowie auch Hellerstein und Shea [6], um einige wichtige Resultate in der Theorie der ganzen und meromorphen Funktionen aufzustellen. Dabei hat Ostrovskiĩ [10] das oben erwähnte Theorem II von Pólya auf folgende Form gebracht:

Satz A. Es seien $h_{1}$ und $h_{2}$ für $t \geqq 0$ reelle Funktionen mit $h_{2}(t) \geqq 0$. Weiter seien zwei Zahlen $\varrho \geqq 0$ und $\varepsilon>0$ so gegeben, daß die beiden Integrale

$$
\int_{0}^{\infty} \frac{\left|h_{1}(t)\right|}{t^{1+\sigma}} d t \quad \text { und } \quad \int_{0}^{\infty} \frac{h_{2}(t)}{t^{1+\sigma}} d t
$$


für $\varrho<\sigma<\varrho+\varepsilon$ konvergieren, aber das zweite Integral für $\sigma<\varrho$ divergiert. Außerdem existiere eine in $\{z:|z-\varrho|<\varepsilon\}$ analytische Funktion $s$, die für reelle $z$ reell ist und die für $\varrho<\sigma<\varrho+\varepsilon$ die Identität

$$
\int_{0}^{\infty} \frac{h_{1}(t)}{t^{1+\sigma}} d t=s(\sigma) \int_{0}^{\infty} \frac{h_{2}(t)}{t^{1+\sigma}} d t
$$

erfüllt.

Unter diesen Voraussetzungen gilt dann die Doppelungleichung

$$
\limsup _{r \rightarrow \infty} \frac{h_{1}(r)}{h_{2}(r)} \geqq s(\varrho) \geqq \liminf _{r \rightarrow \infty} \frac{h_{1}(r)}{h_{2}(r)} .
$$

In der vorliegenden Note werden einige Anwendungen von Satz A gegeben. Dabei werden asymptotische Abschätzungen bewiesen, die mit Ergebnissen des Verfassers [16], [17] und [18] zusammenhängen, und es wird der oben genannte $\cos \pi \varrho$-Satz erweitert.

\section{Ganze Funktionen mit negativen Nullstellen}

\subsection{Es gilt}

Satz 2.1. Ist $f$ ein kanonisches Produkt mit negativen Nullstellen und von nichtganzzahliger Ordnung $\varrho$, so gilt für alle $\theta$ mit $-\pi<\theta \leqq \pi$

$$
\limsup _{r \rightarrow \infty} \frac{\log \left|f\left(r e^{i \theta}\right)\right|}{\bar{N}(r, 0)} \geqq \pi \varrho^{2} \frac{\cos \theta \varrho}{\sin \pi \varrho} \geqq \liminf _{r \rightarrow \infty} \frac{\log \left|f\left(r e^{i \theta}\right)\right|}{\bar{N}(r, 0)} .
$$

Beweis. Ohne Beschränkung der Allgemeinheit kann man $f(0)=1$ setzen. Ist $q=[\varrho]$ das Geschlecht von $f$, so gilt für $\varrho<\sigma<q+1$ (man vergleiche Hellerstein und Shea [6], S. 223)

$$
\int_{0}^{\infty} \frac{\log \left|f\left(t e^{i \theta}\right)\right|}{t^{1+\sigma}} d t=\frac{\pi}{\sigma} \frac{\cos \theta \sigma}{\sin \pi \sigma} \sum_{n=1}^{\infty} \frac{1}{\left|a_{n}\right|^{\sigma}},
$$

wobei $a_{n}$ die Nullstellen von $f$ sind. Beachtet man die Identitäten (man vergleiche Hayman [5], S. 25)

$$
\sum_{n=1}^{\infty} \frac{1}{\left|a_{n}\right|^{\sigma}}=\sigma \int_{0}^{\infty} \frac{n(t, 0)}{t^{1+\sigma}} d t=\sigma^{2} \int_{0}^{\infty} \frac{N(t, 0)}{t^{1+\sigma}} d t=\sigma^{3} \int_{0}^{\infty} \frac{\bar{N}(t, 0)}{t^{1+\sigma}} d t,
$$

so folgt aus (2.2)

$$
\int_{0}^{\infty} \frac{\log \left|f\left(t e^{i \theta}\right)\right|}{t^{1+\sigma}} d t=\pi \sigma^{2} \frac{\cos \theta \sigma}{\sin \pi \sigma} \int_{0}^{\infty} \frac{\bar{N}(t, 0)}{t^{1+\sigma}} d t .
$$


In (2.4) sind alle Voraussetzungen des Satzes $A$ erfüllt, womit dann (2.1) aus (2.4) folgt.

Folgerung. Ist $f$ eine ganze Funktion der Ordnung $\varrho$ mit $0<\varrho<1$, so gilt

$$
\limsup _{r \rightarrow \infty} \frac{\bar{N}(r, 0)}{\log M(r)} \geqq \frac{\sin \pi \varrho}{\pi \varrho^{2}} .
$$

Diese Folgerung ergibt sich sofort aus der rechten Ungleichung von (2.1), wenn man dort $\theta=0$ setzt und dabei beachtet, daß man im Fall $\varrho<1$ ohne Beschränkung der Allgemeinheit die Nullstellen von $f$ als negativ voraussetzen darf.

Ungleichung (2.5) wurde in [18] mit Hilfe einer anderen Methode bewiesen.

2.2. Für ganze Funktionen endlicher Ordnung hat der Verfasser in [16]

$$
\liminf _{r \rightarrow \infty} \frac{N(r, 0)}{v(r)} \leqq \liminf _{r \rightarrow \infty} \frac{\bar{N}(r, 0)}{\log M(r)}
$$

bewiesen. Wir wollen jetzt mit Hilfe von Satz 2.1 folgende Abschätzung der rechten Seite von (2.6) herleiten.

Satz 2.2. Ist $f$ eine ganze Funktion mit negativen Nullstellen und von endlicher Ordnung $\varrho$ sowie vom Geschlecht $q(q \leqq \varrho \leqq q+1)$, so gilt

$$
\liminf _{r \rightarrow \infty} \frac{\bar{N}(r, 0)}{\log M(r)} \leqq \frac{|\sin \pi \varrho|}{\pi \varrho^{2}} .
$$

Beweis. Beim Beweis dieser Ungleichung wird $\varrho>0$ vorausgesetzt.

I. Zuerst wird gezeigt, daß man ohne Beschränkung der Allgemeinheit die ganze Funktion $f$ als kanonisches Produkt voraussetzen darf. Dabei spielt das folgende Ergebnis, das auf Edrei und Fuchs [3] zurückgeht, eine wesentliche Rolle:

Für ein kanonisches Produkt $Q$ mit negativen Nullstellen und vom Geschlecht $q_{1}$ gilt

$$
\lim _{r \rightarrow \infty} \frac{r^{q_{1}}}{\log M(r, Q)}=0 \text {. }
$$

Einen einfachen Beweis von (2.8) findet man in [17].

Geht man von der Produktdarstellung von $f$ aus (man vergleiche Nevanlinna [9], S. 233), so gilt

$$
f(z)=e^{h(z)} Q(z),
$$

wobei $h$ ein Polynom vom Grad $p \leqq q$ und $Q$ ein kanonisches Produkt mit negativen Nullstellen und von der Ordnung $\varrho_{1} \leqq \varrho$ sowie vom Geschlecht $q_{1} \leqq q$ ist. 
A. Es sei $p<\varrho$. Dann gilt bekanntlich notwendig $\varrho_{1}=\varrho$ und damit auch $q_{1}=q$. Daher ergibt sich aus (2.9)

und

$$
\log M(r, f) \leqq K_{1} r^{q_{1}}+\log M(r, Q)
$$

$$
\log M(r, Q) \leqq K_{2} r^{q_{1}}+\log M(r, f),
$$

wobei $K_{1}, K_{2}$ Konstanten sind. Aus diesen beiden Ungleichungen folgt zusammen mit (2.8) sofort

$$
\log M(r, f) \sim \log M(r, Q) .
$$

B. Es sei $p=\varrho$. Ist $\varrho_{1}<\varrho$, so ist auch $\bar{N}(r, 0)$ von der Ordnung $\varrho_{1}$ und man erhält durch elementare Überlegungen

$$
\liminf _{r \rightarrow \infty} \frac{\bar{N}(r, 0)}{\log M(r)}=0 .
$$

Ist $\varrho_{1}=\varrho$, so bleiben die beiden Möglichkeiten $q_{1}=\varrho$ oder $q_{1}=\varrho-1$. Im Fall $q_{1}=\varrho$ zeigt man wie in $\mathrm{A}, \mathrm{da} ß$

$$
\log M(r, f) \sim \log M(r, Q)
$$

ist. Im Fall $q_{1}=\varrho-1$ gilt (man vergleiche Dinghas [2], S. 356)

$$
\log M(r, Q) \leqq K r^{q_{1}+1} \int_{0}^{\infty} \frac{n(t, 0)}{t^{q_{1}+1}} \frac{d t}{t+r},
$$

wobei $K$ eine Konstante ist. Ist $\varepsilon>0$ gegeben, so wähle man ein $x$ derart, daß

$$
\int_{x}^{\infty} \frac{n(t, 0)}{t^{q_{1}+2}} d t<\varepsilon
$$

gilt. Daraus folgt zusammen mit (2.11)

und ferner

$$
\log M(r, Q) \leqq K r^{q_{1}+1}\left\{\frac{1}{r} \int_{0}^{x} \frac{n(t, 0)}{t^{q_{1}+1}} d t+\varepsilon\right\}
$$

$$
\lim _{r \rightarrow \infty} \frac{\log M(r, Q)}{r^{q_{1}+1}}=0 .
$$

Außerdem gilt wegen bekannter Beziehungen (man vergleiche Hayman [5], S. 7, und Nevanlinna [9], S. 168, 172 und 220)

$$
\begin{aligned}
K_{1} r^{q_{1}+1} & =K_{1} r^{p} \leqq T\left(r, e^{h}\right) \leqq T(r, f)+T(r, Q)+K_{2} \\
& \leqq \log M(r, f)+\log M(r, Q)+K_{2},
\end{aligned}
$$

wobei $K_{1}$ und $K_{2}$ Konstanten sind. Aus dieser Ungleichung folgt mit (2.12) für alle $r \geqq r_{0}$

$$
\log M(r, f) \geqq \log M(r, Q) .
$$


Aus A und B folgt das gewünschte Ergebnis, daß man beim Beweis von Satz 2.2 ohne Beschränkung der Allgemeinheit $f$ als kanonisches Produkt voraussetzen darf.

II. Ist $\varrho$ nicht ganzzahlig, so folgt aus der für alle $\theta$ gültigen Ungleichung

$$
\log M(r, f) \geqq \log \left|f\left(r e^{i \theta}\right)\right|
$$

und aus Satz 2.1 mit $\theta=0$ bzw. $\theta=\pi / \varrho$ sofort die gewünschte Abschätzung (2.7).

III. Ist dagegen $\varrho \geqq 1$ ganzzahlig, so greifen wir auf die sogenannte Methode der Pólya peaks zurück, die der Verfasser auch in [18] benutzt hat.

Es gilt (man vergleiche [18], S. 291)

$$
\log M(r) \geqq \frac{1}{2} r^{q+1} \int_{0}^{\infty} \frac{\bar{N}(t, 0)}{t^{q+1}} \frac{q^{2}(t+r)^{2}+2 q t(t+r)+t^{2}-t r}{(t+r)^{3}} d t .
$$

Man nehme als Antithese an, daß

$$
\liminf _{r \rightarrow \infty} \frac{\bar{N}(r, 0)}{\log M(r)}=\alpha>0
$$

gilt.

Sei zunächst $\alpha<+\infty$. Dann existiert zu jedem $\varepsilon>0$ ein $r_{0}$ derart, daß

$$
\bar{N}(r, 0) \geqq(\alpha-\varepsilon) \log M(r)
$$

für alle $r \geqq r_{0}$ gilt. Da der Integrand in (2.13) positiv ist, folgt mit (2.14) für $r \geqq r_{0}$

$$
\log M(r) \geqq \frac{1}{2}(\alpha-\varepsilon) r^{q+1} \int_{r_{0}}^{\infty} \frac{\log M(t)}{t^{q+1}} \frac{q^{2}(t+r)^{2}+2 q t(t+r)+t^{2}-t r}{(t+r)^{3}} d t .
$$

Daraus ergibt sich mit einem Hilfssatz über Pólya peaks (man vergleiche [18]) für die Folgen $\left(r_{n}\right),\left(a_{n}\right)$ und $\left(A_{n}\right)$ mit

$\log M\left(r_{n}\right)$

$$
\lim _{n \rightarrow \infty} a_{n}=\lim _{n \rightarrow \infty} \frac{A_{n}}{r_{n}}=\lim _{n \rightarrow \infty} \frac{r_{n}}{a_{n}}=\infty:
$$

$$
\geqq \frac{1}{2}(\alpha-\varepsilon)(1+o(1)) \log M\left(r_{n}\right) \int_{a_{n}}^{A_{n}}\left(\frac{t}{r_{n}}\right)^{e-q-1} \frac{q^{2}\left(t+r_{n}\right)^{2}+2 q t\left(t+r_{n}\right)+t^{2}-t r_{n}}{\left(t+r_{n}\right)^{3}} d t .
$$

Ist nun $\varrho$ ganzzahlig, also $\varrho=q$ oder $\varrho=q+1$, so strebt mit $n \rightarrow \infty$ das Integral gegen unendlich, und man erhält einen Widerspruch $z u \quad \alpha>0$.

Für $\alpha=+\infty$ geht der Beweis analog.

Bemerkung. Mit der gleichen Methode beweist man für ganze Funktionen mit negativen Nullstellen und von ganzzahliger Ordnung (man vergleiche dazu [17])

$$
\liminf _{r \rightarrow \infty} \frac{n(r, 0)}{\log M(r)}=\liminf _{r \rightarrow \infty} \frac{N(r, 0)}{\log M(r)}=0 .
$$


2.3. In [15] hat der Verfasser das asymptotische Verhalten von $n(r, 0) / T(r)$ untersucht und für ganze Funktion der Ordnung $\varrho<1$ gezeigt

$$
\limsup _{r \rightarrow \infty} \frac{n(r, 0)}{T(r)} \geqq \begin{cases}\varrho, & 0 \leqq \varrho \leqq \frac{1}{2} \\ \varrho \sin \pi \varrho, & \frac{1}{2} \leqq \varrho<1 .\end{cases}
$$

Hier wird nun eine Abschätzung in umgekehrter Richtung gegeben.

Satz 2.3. Ist $f$ eine ganze Funktion mit negativen Nullstellen und von der nicht ganzzahligen Ordnung $\varrho$ sowie vom Geschlecht $q=[\varrho]$, so gilt

$$
\begin{gathered}
\liminf _{r \rightarrow \infty} \frac{n(r, 0)}{T(r)} \leqq \begin{cases}\frac{\varrho|\sin \pi \varrho|}{q+|\sin \pi \varrho|}, & q<\varrho \leqq q+\frac{1}{2} \\
\frac{\varrho|\sin \pi \varrho|}{q+1}, & q+\frac{1}{2} \leqq \varrho<q+1,\end{cases} \\
\liminf _{r \rightarrow \infty} \frac{\bar{N}(r, 0)}{T(r)} \leqq \begin{cases}\frac{1}{\varrho} \frac{|\sin \pi \varrho|}{q+|\sin \pi \varrho|}, & q<\varrho \leqq q+\frac{1}{2} \\
\frac{1}{\varrho} \frac{|\sin \pi \varrho|}{q+1}, & q+\frac{1}{2} \leqq \varrho<q+1 .\end{cases}
\end{gathered}
$$

Beweis. Aus (2.8) und der bekannten Ungleichung (Nevanlinna [9], S. 220)

$$
\log M(r) \leqq \frac{R+r}{R-r} T(R)
$$

für $R>r$, folgt für kanonisches Produkt mit negativen Nullstellen vom Geschlecht $q$

$$
\lim _{r \rightarrow \infty} \frac{r^{q}}{T(r)}=0 .
$$

Damit zeigt man nun leicht, daß man ohne Beschränkung der Allgemeinheit $f$ als kanonisches Produkt voraussetzen darf.

Wegen (2.2) und (2.3) gilt für $\varrho<\sigma<q+1$

$$
\begin{aligned}
\int_{0}^{\infty} \frac{\log \left|f\left(t e^{i \theta}\right)\right|}{t^{1+\sigma}} d t & =\pi \frac{\cos \theta \sigma}{\sin \pi \sigma} \int_{0}^{\infty} \frac{n(t, 0)}{t^{1+\sigma}} d t \\
& =\pi \sigma^{2} \frac{\cos \theta \sigma}{\sin \pi \sigma} \int_{0}^{\infty} \frac{\bar{N}(t, 0)}{t^{1+\sigma}} d t .
\end{aligned}
$$

Setzt man

mit

$$
\tilde{m}_{e}(r, f)=\int_{E_{Q}} \log \left|f\left(r e^{i \theta}\right)\right| d \theta
$$

$$
E_{\varrho}=\left\{\theta \mid-\pi<\theta<\pi \text { und } \frac{\cos \theta \varrho}{\sin \pi \varrho}>0\right\},
$$


so folgt aus (2.18)

$$
\begin{aligned}
\int_{0}^{\infty} \frac{\tilde{m}_{\varrho}(t, f)}{t^{1+\varrho}} d t & =\frac{\pi}{\sin \pi \sigma} \int_{E_{\varrho}} \cos \theta \sigma d \theta \int_{0}^{\infty} \frac{n(t, 0)}{t^{1+\sigma}} d t \\
& =\frac{\pi \sigma^{2}}{\sin \pi \sigma} \int_{E_{\varrho}} \cos \theta \sigma d \theta \int_{0}^{\infty} \frac{\bar{N}(t, 0)}{t^{1+\sigma}} d t .
\end{aligned}
$$

Wendet man auf (2.19) Satz A an, so ergibt sich

$$
\limsup _{r \rightarrow \infty} \frac{\tilde{m}_{\varrho}(r, f)}{n(r, 0)} \geqq \frac{\pi}{\sin \pi \varrho} \int_{E_{Q}} \cos \theta \varrho d \theta \geqq \liminf _{r \rightarrow \infty} \frac{\tilde{m}_{\varrho}(r, f)}{n(r, 0)}
$$

und die entsprechende Ungleichung für $\bar{N}(r, 0)$. Wegen

folgt aus (2.20)

$$
\tilde{m}_{\varrho}(r, f) \leqq 2 \pi T(r)
$$

$$
\limsup _{r \rightarrow \infty} \frac{T(r)}{n(r, 0)} \geqq \frac{1}{2 \sin \pi \varrho} \int_{E_{\varrho}} \cos \theta \varrho d \theta
$$

und daraus durch elementare Rechnungen (2.16).

Entsprechend ergibt sich (2.17).

Bemerkung. Mit Hilfe einer Ungleichung von Shea, die man bei Hellerstein und Williamson [7], S. 335, findet, läßt sich (2.15) sofort auf meromorphe Funktionen übertragen. Für meromorphe Funktionen der Ordnung $\varrho<1$ hat die Abschätzung dann die Gestalt

$$
\limsup _{r \rightarrow \infty} \frac{n(r, 0)+n(r, \infty)}{T(r)} \geqq \begin{cases}\varrho, & 0 \leqq \varrho \leqq \frac{1}{2} \\ \varrho \sin \pi \varrho, & \frac{1}{2} \leqq \varrho<1\end{cases}
$$

\section{Ganze Funktionen mit negativen Nullstellen der Ordnung $\varrho<1$}

3.1. Bekanntlich genügt es, den $\cos \pi \varrho$-Satz für ganze Funktionen mit negativen Nullstellen zu beweisen (man vergleiche z. B. Boas [1], S. 40). Dann besitzt aber die ganze Funktion $f$ die Darstellung

$$
f(z)=\prod_{n=1}^{\infty}\left(1+\frac{z}{r_{n}}\right) \quad\left(r_{n}>0\right) .
$$

Für diese Funktion gilt nun

$$
M(r)=|f(r)| \quad \text { und } \quad m(r)=|f(-r)|,
$$


und daher läßt sich der $\cos \pi \varrho-S a t z$ in der Form

$$
\limsup _{r \rightarrow \infty} \frac{\log |f(-r)|}{\log |f(r)|} \geqq \cos \pi \varrho
$$

schreiben. Diese Form des $\cos \pi \varrho$-Satzes gibt Anlaß dazu, die Frage des asymptotischen Verhaltens von

$$
\frac{\log \left|f\left(r e^{i \theta}\right)\right|}{\log \left|f\left(r e^{i \varphi}\right)\right|}
$$

$\mathrm{zu}$ behandeln.

Satz 3.1. Ist $f$ eine ganze Funktion mit negativen Nullstellen der Ordnung $\varrho$ mit $0<\varrho<1$, so gilt für $-\pi<\theta \leqq \pi$ und $-\pi / 2 \leqq \varphi \leqq \pi / 2$

$$
\limsup _{r \rightarrow \infty} \frac{\log \left|f\left(r e^{i \theta}\right)\right|}{\log \left|f\left(r e^{i \varphi}\right)\right|} \geqq \frac{\cos \theta \varrho}{\cos \varphi \varrho} \geqq \liminf _{r \rightarrow \infty} \frac{\log \left|f\left(r e^{i \theta}\right)\right|}{\log \left|f\left(r e^{i \varphi}\right)\right|} .
$$

Beweis. A. Es folgt aus (2.2) für $\varrho<\sigma<1$

$$
\int_{0}^{\infty} \frac{\log \left|f\left(t e^{i \theta}\right)\right|}{t^{1+\sigma}} d t=\frac{\cos \theta \sigma}{\cos \varphi \sigma} \int_{0}^{\infty} \frac{\log \left|f\left(t e^{i \varphi}\right)\right|}{t^{1+\sigma}} d t .
$$

Benutzt man Produktdarstellung (3.1) von $f$, so erkennt man, daß $\log \left|f\left(r e^{i \varphi}\right)\right| \geqq 0$ für $-\pi / 2 \leqq \varphi \leqq \pi / 2$ gilt. Zeigt man noch für $\mu<\varrho$ und $-\pi / 2 \leqq \varphi \leqq \pi / 2$

$$
\int_{0}^{\infty} \frac{\log \left|f\left(t e^{i \varphi}\right)\right|}{t^{1+\mu}} d t=+\infty
$$

so folgt (3.2) aus (3.3) und Satz A.

B. Für $-\pi<\varphi<\pi$ gilt (man vergleiche Titchmarsh [13], S. 271)

$$
\log f\left(r e^{i \varphi}\right)=r e^{i \varphi} \int_{0}^{\infty} \frac{n(t, 0)}{t\left(t+r e^{i \varphi}\right)} d t .
$$

Daraus ergibt sich für $-\pi / 2 \leqq \varphi \leqq \pi / 2$

$$
\begin{aligned}
\log \left|f\left(r e^{i \varphi}\right)\right| & =r \int_{0}^{\infty} \frac{n(t, 0)}{t} \frac{t \cos \varphi+r}{t^{2}+r^{2}+2 t r \cos \varphi} d t \\
& \geqq r \int_{r}^{\infty} \frac{n(t, 0)}{t} \frac{r}{(t+r)^{2}} d t \\
& \geqq n(r, 0) \int_{1}^{\infty} \frac{d s}{s(1+s)^{2}}=n(r, 0) K,
\end{aligned}
$$

wobei $K>0$ eine Konstante ist. Da bekanntlich auch $n(r, 0)$ von der Ordnung $\varrho$ ist, folgt (3.4) aus (3.5), womit Satz 3.1 bewiesen ist. 
Bemerkungen. Setzt man in (3.2) $\theta=\pi$ und $\varphi=0$, so erhält man gerade den klassischen $\cos \pi \varrho$-Satz.

Für $\theta=\pi$ ist die rechte Seite der Ungleichung (3.2) sicher nicht scharf. Ist aber $-\pi<\alpha<\pi$ und $n(r, 0) \sim r^{o}$, so gilt (man vergleiche Boas [1], S. 55)

also

$$
\log f\left(r e^{i \alpha}\right) \sim e^{i \varrho \alpha} \frac{\pi r^{\varrho}}{\sin \pi \varrho},
$$

und daher

$$
\log \left|f\left(r e^{i \alpha}\right)\right| \sim \pi r^{\varrho} \frac{\cos \alpha \varrho}{\sin \pi \varrho}
$$

$$
\frac{\log \left|f\left(r e^{i \theta}\right)\right|}{\log \left|f\left(r e^{i \varphi}\right)\right|} \sim \frac{\cos \theta \varrho}{\cos \varphi \varrho}
$$

für $-\pi<\theta, \varphi<\pi$. Damit ist (3.2) für $\theta \neq \pi$ nicht zu verbessern.

3.2. Im folgenden setze man für $0<\beta \leqq \pi$

$$
m(r, f, \beta)=\frac{1}{\pi} \int_{0}^{\beta} \log \left|f\left(r e^{i \theta}\right)\right| d \theta .
$$

Satz 3.2. Ist $f$ eine ganze Funktion mit negativen Nullstellen der Ordnung $\varrho$ mit $0<\varrho<1$, so gilt für $-\pi / 2 \leqq \varphi \leqq \pi / 2$

$$
\limsup _{r \rightarrow \infty} \frac{m(r, f, \beta)}{\log \left|f\left(r e^{i \varphi}\right)\right|} \geqq \frac{1}{\pi \varrho} \frac{\sin \varrho \beta}{\cos \varrho \varphi} \geqq \liminf _{r \rightarrow \infty} \frac{m(r, f, \beta)}{\log \left|f\left(r e^{i \varphi}\right)\right|} .
$$

Beweis. Für $\varrho<\sigma<1$ folgt aus (2.2)

$$
\int_{0}^{\infty} \frac{m(t, f, \beta)}{t^{1+\sigma}} d t=\frac{1}{\sigma^{2}} \frac{\sin \beta \sigma}{\sin \pi \sigma} \sum_{n=1}^{\infty} \frac{1}{r_{n}^{\sigma}}
$$

und daraus zusammen mit (2.2)

$$
\int_{0}^{\infty} \frac{m(t, f, \beta)}{t^{1+\sigma}} d t=\frac{1}{\pi \sigma} \frac{\sin \beta \sigma}{\cos \varphi \sigma} \int_{0}^{\infty} \frac{\log \left|f\left(t e^{i \varphi}\right)\right|}{t^{1+\sigma}} d t .
$$

Beachtet man den Beweis von Satz 3.1 so erkennt man, daß alle Voraussetzungen von Satz A erfüllt sind, und daher ergibt sich aus der Identität (3.7) die Aussage des Satzes 3.2.

Aus Satz 3.2 folgt leicht die Vermutung von Paley [11] für ganze Funktionen mit negativen Nullstellen der Ordnung $0<\varrho<1$

$$
\limsup _{r \rightarrow \infty} \frac{T(r)}{\log M(r)} \geqq \begin{cases}\frac{\sin \pi \varrho}{\pi \varrho}, & 0<\varrho \leqq \frac{1}{2} \\ \frac{1}{\pi \varrho}, & \frac{1}{2} \leqq \varrho<1 .\end{cases}
$$


Denn da die Nullstellen von $f$ negativ sind, ist $\left|f\left(r e^{i \theta}\right)\right|$ eine gerade Funktion von $\theta$ und daher gilt für alle $\beta$ mit $0<\beta \leqq \pi$

$$
T(r)=\frac{1}{\pi} \int_{0}^{\pi} \log ^{+}\left|f\left(r e^{i \theta}\right)\right| d \theta \geqq m(r, f, \beta) .
$$

Daraus und aus (3.6) mít $\varphi=0$ und $\beta=\pi$ für $\varrho \leqq 1 / 2$ sowie mit $\beta=\pi /(2 \varrho)$ für $\varrho \geqq 1 / 2$ folgt Ungleichung (3.8).

Die Vermutung von Paley ist für alle ganzen Funktionen richtig. Den ersten vollständigen Beweis dafür gab Govorov [4].

\section{Literatur}

[1] BoAs, R. P., JR.: Entire functions. - Pure and Applied Mathematics 5. Academic Press Inc., New York, 1954.

[2] Dinghas, A.: Vorlesungen über Funktionentheorie. - Die Grundlehren der mathematischen Wissenschaften 110. Springer-Verlag, Berlin-Göttingen-Heidelberg, 1961.

[3] EdReI, A., und W. H. J. Fuchs: On the growth of meromorphic functions with several deficient values. - Trans. Amer. Math. Soc. 93, 1959, 292-328.

[4] Govorov, N. V.: Paley's hypothesis. - Functional Anal. Appl. 3, 1969, 115-118. [Translation from: Funkcional. Anal. i Priložen. 3:2, 1969, 41-45.]

[5] Hayman, W. K.: Meromorphic functions. - Oxford Mathematical Monographs. The Clarendon Press, Oxford, 1964.

[6] Hellerstein, S., und D. F. Shea: Bounds for the deficiencies of meromorphic functions of finite order. - Entire functions and related parts of analysis. Proceedings of Symposia in Pure Mathematics 11. American Mathematical Society, Providence (Rhode Island), 1968, 214-239.

[7] Hellerstein, S., und J. Williamson: Entire functions with negative zeros and a problem of R. Nevanlinna. - J. Analyse Math. 22, 1969, 233-267.

[8] Littlewood, J. E.: A general theorem on integral functions of finite order. - Proc. London Math. Soc. (2) 6, 1908, 189-204.

[9] Nevanlinna, R.: Eindeutige analytische Funktionen. - [Zweite verbesserte Auflage.] Die Grundlehren der mathematischen Wissenschaften 46. Springer-Verlag, Berlin-Göttingen-Heidelberg, 1953.

[10] OstrovskIĬ, I. V. [И. В. Островский]: О некоторых асимптотических свойствах целых функций с вещественными отрицательными нулями. - Zap. Meh.-Mat. Fak. i Har'kov. Mat. Obšč. (4) 28, 1961, 23-32.

[11] Paley, R. E. A. C.: A note on integral functions. - Proc. Cambridge Philos. Soc. 28, 1932, 262-265.

[12] Pólya, G.: On the minimum modulus of integral functions. - J. London Math. Soc. 1, 1926, $78-86$.

[13] Titchmarsh, E. C.: The theory of functions. - [Second edition.] Oxford University Press, London, 1939.

[14] Valiron, G.: Sur les fonctions entières d'ordre nul et d'ordre fini et en particulier les fonctions à correspondance régulière. - Ann. Fac. Sci. Univ. Toulouse (3) 5, 1913 [1914], $117-257$. 
[15] VolkmanN, L.: Über einen Zusammenhang zwischen der charakteristischen Funktion und der Anzahl der Nullstellen bei ganzen Funktionen der Ordnung $\lambda<1$. - An. Şti. Univ. “Al. I. Cuza” Iaşi Secț. I a Mat. 20, 1974, 305-308.

[16] VolkmanN, L.: Über Zusammenhänge zwischen den Nullstellen und dem Zentralindex ganzer Funktionen. - Math. Ann. 217, 1975, 87-91.

[17] Volkmann, L.: Ganze Funktionen mit negativen Nullstellen. - Math. Scand. 40, 1977, 143150.

[18] VolkmanN, L.: Zusammenhänge zwischen dem Maximalbetrag und einer Nullstellenfunktion bei ganzen Funktionen. - Arch. Math. (Basel) 30, 1978, 286-292.

[19] Wiman, A.: Über eine Eigenschaft der ganzen Funktionen von der Höhe Null. - Math. Ann. 76, 1915, 197-211.

Freie Universität Berlin

I. Mathematisches Institut

Hüttenweg 9

D 1000 Berlin 33

Eingegangen am 27. Februar 1979 